\title{
Journal of Attention Disorders
}

http://jad.sagepub.com

\section{Ethnic Differences in Parental Beliefs of Attention-Deficit/Hyperactivity Disorder and Treatment Andy V. Pham, John S. Carlson and John F. Kosciulek \\ J Atten Disord 2010; 13; 584 originally published online May 4, 2009; \\ DOI: $10.1177 / 1087054709332391$}

The online version of this article can be found at: http://jad.sagepub.com/cgi/content/abstract/13/6/584

\author{
Published by: \\ (-) SAGE \\ http://www.sagepublications.com
}

Additional services and information for Journal of Attention Disorders can be found at:

Email Alerts: http://jad.sagepub.com/cgi/alerts

Subscriptions: http://jad.sagepub.com/subscriptions

Reprints: http://www.sagepub.com/journalsReprints.nav

Permissions: http://www.sagepub.com/journalsPermissions.nav

Citations http://jad.sagepub.com/cgi/content/refs/13/6/584 
and Treatment

\author{
Andy V. Pham \\ John S. Carlson \\ John F. Kosciulek \\ Michigan State University
}

\begin{abstract}
Objective: A survey study was conducted to explore ethnic differences in parental beliefs about the causes and treatments of ADHD and whether these beliefs predicted treatment preference. Method: Ethnically diverse parents of 5- to 12-year-old children with ADHD $(n=58)$ and without ADHD $(n=61)$ completed a questionnaire developed by the authors that asked them to rate statements about biological and psychological causes of ADHD and their beliefs about medication and behavioral treatment. Results: There were no significant ethnic differences in how parents viewed causes of ADHD. Beliefs about behavioral treatment revealed significant group differences, as ethnic minority (e.g., African American, Latino) parents rated behavioral treatments more positively than did Caucasian parents. Beliefs about biological causes predicted medication treatment and combined treatment use. Conclusion: Dissemination of information regarding evidence-based treatments should be given special attention as it may influence parents' decisions to pursue specific treatments based on their beliefs. (J. of Att. Dis. 2010; 13(6) 584-591)
\end{abstract}

Keywords: ADHD; parental beliefs; ethnic minorities; treatment use; survey

$\mathrm{R}$ educing mental health disparities of ethnic minority families has always been a challenging task for researchers and practitioners. Despite the many services available for children with ADHD, research suggests that ethnic minority families (e.g., African American, Latino) are less likely to use these services compared with Caucasian families (Bailey, 2005; Eiraldi, Mazzuca, Clarke, \& Power, 2006; Rothe, 2005). One potential reason for this disparity in service utilization is that different cultural beliefs about the causes of mental health and behavioral problems, such as ADHD, may influence how and whether ethnic minority families seek help. Because families may have different explanations about the nature of problem behaviors than practitioners do, they may pursue alternative forms of treatment or decide not to pursue treatment at all (Eiraldi et al., 2006). Unfortunately, few studies have addressed how multiethnic samples experience ADHD (Perry, Hatton, \& Kendall, 2005). There has been little cross-cultural research exploring how parents from ethnic minority backgrounds perceive symptoms of ADHD, their beliefs about the causes and treatments of the disorder, and how these parental perceptions influence the course of treatment (Johnston, Seipp, Hommersen, Hoza, \& Fine, 2005). Understanding these cultural factors is necessary because of the parents' important role in selecting and accessing treatments and service providers for children with ADHD (Bussing, Zima, \& Belin, 1998; Taylor \& Leitman, 2003).

Empirically validated treatment approaches for ADHD include an array of psychotropic medication, psychosocial interventions, or a combination of both. Of all the pharmacological treatments available for children and adolescents, stimulant medication (e.g., methylphenidate) has been the most meticulously investigated and employed,

\footnotetext{
Authors' Note: Address correspondence to Andy V. Pham, Michigan State University, School Psychology Program, 435 Erickson Hall, East Lansing, MI 48824; e-mail: phamandy@msu.edu.
} 
primarily for managing ADHD (Brown, 2005). Although stimulants have been found to improve children's abilities to attend and to decrease hyperactivity via biochemical changes, psychosocial treatments for ADHD have been found to improve social and interpersonal relationships with peers and parents. Examples of evidence-based psychosocial treatments include parent-training or teachertraining programs, which primarily use operant procedures and contingency management in reducing ADHD-related behaviors in children. Medication can be successfully combined with psychosocial interventions for children with severe behavioral problems or comorbid psychiatric conditions (Majewicz-Hefley \& Carlson, 2007). However, there are current health system barriers that prevent many families, in particular ethnic minority families, from seeking and utilizing evidence-based treatment. These barriers include the lack of culturally competent health care providers, financial barriers, and little dissemination of information about ADHD treatment to families (Bailey \& Owens, 2005).

\section{Parental Beliefs of ADHD and Treatment}

Research regarding the etiology of ADHD suggests that an interplay of biological and psychosocial factors leads to a final biopsychosocial pathway expressed as ADHD (Rapport, Chung, Shore, \& Isaacs, 2001). However, Latino parents and African American parents were generally less likely than Caucasian parents to endorse causes consistent with biopsychosocial beliefs about mental illness (Yeh, Hough, McCabe, Lau, \& Garland, 2004). If certain parents have different explanations about their children's problems that are less consistent with biopsychosocial causes, they would be less likely to accept biopsychosocial mental health services.

Bussing, Gary, Mills, and Garvan (2003) interviewed African American and Caucasian parents of children with ADHD to examine whether parents' understanding of the disorder contributed to cultural variations in parental help-seeking behavior. Caucasian parents were more likely to use medical terminology in describing ADHD than African American parents. African American parents were less likely to connect the school system to ADHD problem identification and expressed fewer worries about ADHD-related school problems than did Caucasian parents. This finding raises questions about whether school factors play a role in the reduced access to professional ADHD services for African American families (Bussing et al., 2003). In addition, more African American parents were not sure about potential causes of and treatments for ADHD and were less likely to request medication treatment and school-based interventions. These results suggest a greater need for services involving culturally appropriate parent education within the school and mental health communities.

Parental expectations and attitudes toward mental health services for ADHD are thought to have a significant impact on the parents' decision to seek help for their children (Eiraldi et al., 2006). dosReis and colleagues (2003) examined parental knowledge and perceptions of stimulant medication treatment for ADHD. Perceptions of stimulant medication differed between Caucasian and ethnic minority parents (e.g., African American, Latino) who had children diagnosed with ADHD. Compared to Caucasian parents, ethnic minority parents were less likely to recommend medication, were less likely to prefer medication over counseling, and tended to be less satisfied with medication over all. On the other hand, they were more likely than Caucasian parents to believe that medication use leads to drug abuse and adverse side effects (dosReis et al., 2003).

The purpose of the study is to explore cultural differences in parental beliefs of the causes of ADHD and treatment and whether these cognitions influenced treatment preference. As a step to increase cultural awareness among researchers, physicians, psychologists, and other practitioners regarding the needs of ethnic minority populations, an effort must be made to explore cultural beliefs as they relate to treatment acceptability and utilization. Determining the level of unmet needs for mental health services for ethnic minority children with ADHD is necessary for resource allocation and intervention. Therefore, by understanding parental beliefs pertaining to the causes and treatments of the disorder, this knowledge would assist researchers and practitioners to develop school- and community-based programs for the purpose of addressing the needs of families who have concerns about ADHD.

The research questions for the study were as follows: (a) Are there significant ethnic differences in parental beliefs about the etiology of ADHD (i.e., biological causes vs. psychological causes vs. biopsychosocial causes)? (b) Are there significant ethnic differences in how parents view various treatments for ADHD (i.e., medication vs. counseling or behavioral treatment vs. combined treatment)? (c) What is the relationship between parental beliefs about the etiology of ADHD and treatment preferences? (d) What is the relationship between how parents view various treatments of ADHD and their treatment preferences? 


\section{Method}

\section{Participants}

A total of 137 parents were recruited for the study. However, data of 119 parents were used because they completed the survey in its entirety. In all, 99 parents $(83.2 \%)$ who completed the survey were female, and 64 parents $(53.8 \%)$ were of ethnic majority (i.e., White or Caucasian) and 55 parents $(46.2 \%)$ were of ethnic minority backgrounds (i.e., African American, Latino American). A total of 58 parents (48.7\%) reported having a child with ADHD. Of those parents, 52 parents reported that they had a child who was diagnosed with ADHD combined type, 4 parents reported their child being diagnosed with ADHD predominantly hyperactive/ impulsive type, and 2 parents reported their child being diagnosed with ADHD predominantly inattentive type. Parents also reported their child's age from 5 to 12; the average age reported was 9 years. A total of 95 parents $(79.8 \%)$ reported their child was male.

\section{Instruments}

A questionnaire based on previous surveys exploring beliefs and knowledge of ADHD and treatment was created to fit the purpose and research questions for the study. Three measures were used in designing the questionnaire: the ADHD Beliefs and Attitudes Scale (Johnston, 2001), ADHD Knowledge and Opinions Scale-Revised (Rostain, Power, \& Atkins, 1993), and the Attention Deficit Hyperactivity Disorder and Treatment (Stroh, Frankenberger, \& Cornell-Swanson, 2006). The questionnaire gathered responses on a 5-point Likert-type scale (e.g., $1=$ strongly disagree, $5=$ strongly agree) to a total of 36 statements that relate to how participants view the causes and treatments of ADHD. A collaboration of university faculty and students reviewed the questionnaire for content. The questionnaire was piloted with a group of parents in Michigan to determine the length of time needed to complete the survey, the clarity of the questions, and content validity. Changes in the questionnaire included simplifying wording of statements for parents (e.g., the term counseling is used for the items in the questionnaire because of parents' greater familiarity with that term than behavioral treatment) and reducing the number of items so participants would be able to complete the questionnaire within a 15-min time period.

A principal components analysis on the 36 items of the scale was conducted to determine internal reliability and how variables are related to underlying constructs. A four-factor solution was produced, which accounted for $48 \%$ of the variance in scores. The first factor was defined as Beliefs of Biological/Genetic Causes, and the highest loading items included "ADHD is caused by a chemical imbalance in the brain" (.60) and "ADHD is inherited or passed along in the family" (.59). The second factor was defined as Beliefs of Behavioral/ Psychological Causes, and the highest loading items were "ADHD results from parents being inconsistent with rules and consequences" (.61) and "The amount of structure in the child's environment (e.g., routines) can affect ADHD symptoms" (.55). The third factor was labeled as Beliefs Of Medication Treatment, and the highest loading items were "Medication is an effective treatment for ADHD" (.58) and "Medication for ADHD is safe and has only minor side effects" (.57). The final factor was labeled as Beliefs of Behavioral Treatment, and the highest loading items included "Clear, consistent rules and consequences are helpful for children with ADHD" (.71) and "Behavior therapy or counseling is an effective treatment for ADHD" (.63). The factors each had moderate internal consistency (Beliefs of Biological/ Genetic Causes, 6 items, $\alpha=.61$; Beliefs of Behavioral/ Psychological Causes, 8 items, $\alpha=.55$; Beliefs of Medication Treatment, 14 items, $\alpha=.64$; Beliefs of Behavioral Treatment, 8 items, $\alpha=.53$ ). The number of moderate loading marker items and the internal consistencies for the scales supported the appropriateness of the principal components analysis despite the small sample size.

Two open-response questions were provided to elicit qualitative information on the participants' beliefs of the causes of ADHD and their treatment preference. Participants were asked to report which treatment their child with ADHD was currently taking and their reasons for their selection. If participants did not have a child diagnosed with ADHD, they were given a hypothetical situation in which their child did have ADHD. They were still required to select their preferred method of treatment and provide their reasons for their selection. The second open-response question directly asked participants their own beliefs about the causes of ADHD.

\section{Variables}

In this study, parental beliefs were defined as perceived knowledge, attitudes, and opinions about the causes of ADHD and treatment. Beliefs about the causes, or etiology, of ADHD were divided into two categories: beliefs of biological/genetic causes and beliefs of behavioral/psychological causes. Parents who believe 
Table 1

Mean Scores and Standard Deviations of Parental Beliefs by Ethnicity and ADHD Diagnosis

\begin{tabular}{|c|c|c|c|c|c|c|c|c|}
\hline & \multicolumn{2}{|c|}{$\begin{array}{c}\text { Biological or } \\
\text { Genetic Causes }\end{array}$} & \multicolumn{2}{|c|}{$\begin{array}{c}\text { Behavioral or } \\
\text { Psychological Causes }\end{array}$} & \multicolumn{2}{|c|}{$\begin{array}{l}\text { Medication } \\
\text { Treatment }\end{array}$} & \multicolumn{2}{|c|}{$\begin{array}{c}\text { Counseling or } \\
\text { Behavioral Treatment }\end{array}$} \\
\hline & $M$ & $S D$ & $M$ & $S D$ & $M$ & $S D$ & $M$ & $S D$ \\
\hline Ethnic majority (Caucasian) & 2.98 & 0.29 & 2.23 & 0.27 & 3.02 & 0.22 & $3.52 *$ & 0.36 \\
\hline Child with ADHD & 3.07 & 0.31 & 2.17 & 0.23 & 3.03 & 0.23 & 3.56 & 0.42 \\
\hline Child without ADHD & 2.92 & 0.27 & 2.27 & 0.29 & 3.01 & 0.21 & 3.50 & 0.31 \\
\hline Child without ADHD & 2.82 & 0.37 & 2.36 & 0.46 & 2.97 & 0.30 & 3.62 & 0.26 \\
\hline African American & 2.92 & 0.40 & 2.17 & 0.44 & 2.96 & 0.30 & 3.76 & 0.32 \\
\hline Latino American & 2.97 & 0.35 & 2.21 & 0.46 & 2.93 & 0.27 & 3.73 & 0.36 \\
\hline
\end{tabular}

Note: Ratings are from 1 (strongly disagree) to 5 (strongly agree).

$* p<.001$.

that ADHD is attributed to biological factors perceive that the disorder is because of changes in brain activity, neurological functioning, or a genetic predisposition. Behavioral or psychological causes were described as factors within the child's social environment, such as parenting skills, structure of environment, and stress, which can also influence the course of the disorder.

Beliefs about treatment were divided into two categories: beliefs of medication treatment and beliefs of behavioral treatment. Medication treatment includes active administration of current medication (e.g., Ritalin) or other forms of psychotropic medication that are prescribed for children with ADHD. beliefs of behavioral treatment includes psychosocial interventions that address the child's behavior through parent-training programs, counseling, social skills programs, or behavioral management. The term counseling was used for the items in the questionnaire because of parents' greater familiarity with the term than behavioral treatment (Rostain et al., 1993). Use of combined treatment methods likely suggests that parents may believe in a biopsychosocial treatment approach for ADHD. Therefore, mean scores were calculated to produce an additional variable, beliefs of biopsychosocial treatment, if parents' mean scores for both beliefs of medication treatment and beliefs of behavioral treatment were greater than 3.0.

The criterion variable in this study is the parent's treatment preference. Treatment preference is defined as the parents' choice and acceptability of a treatment method that they would select if they have a child diagnosed with ADHD. For this study, parents could select the following treatments: only medication, only counseling, and combined treatment. Parents' beliefs about the causes of ADHD and their beliefs about the varied treatments (i.e., predictor variables) may have an influence on their preferred choice of treatment.

\section{Procedures}

Flyers announcing the research study were posted in mid-Michigan, primarily in educational, community, and health care institutions that serve parents and children with ADHD. Copies of the questionnaire along with an informed consent letter were developed into a packet for distribution. Data were collected at three specific sites: a Head Start program, a rehabilitation clinic, and a local community center. Participants indicated their voluntary participation in the study by signing a consent form. Participants also received monetary reimbursement in the form of a $\$ 10$ gift card for their time and effort in completing the questionnaires.

\section{Results}

Table 1 presents the descriptive statistics for parents' scores on each of the four belief categories by ethnicity and also whether parents have a child who was diagnosed with ADHD. Scores were averaged and divided by the total number of items in each category. Across each ethnic group, parents rated beliefs of biological/genetic causes of ADHD consistently higher than beliefs of behavioral/psychological causes. Each group also rated beliefs of behavioral treatment for ADHD more positively than beliefs of medication treatment.

An analysis of variance was used to assess differences between ethnic groups (i.e., ethnic majority vs. ethnic minority) on parental beliefs of ADHD causes (i.e., biological or genetic causes vs. behavioral or psychological 
causes). Results indicate that there were no significant differences between the two ethnic groups on beliefs of biological/genetic causes, $F(1,117)=0.362, p=.529$, and beliefs of behavioral/psychological causes, $F(1,117)=$ $0.403, p=.527$. Qualitative data also suggested little to no ethnic differences in how parents view causes of ADHD. Ethnic majority parents had a similar number of responses in relation to a biological view, such as "ADHD is caused by a chemical imbalance in the brain" ( 24 responses), when compared with ethnic minority parents (23 responses). There were no significant differences between ethnic groups on beliefs of medication treatment, $F(1$, $117)=2.35, p=.128$; however, there was a significant difference between ethnic groups in beliefs of behavioral treatment, $F(1,117)=6.64, p<.001$. Ethnic minority parents rated counseling and behavioral treatments of ADHD more positively than Caucasian parents.

An analysis of covariance was conducted to assess ethnic differences in beliefs of medication treatment and behavioral treatment by controlling for child's age, gender, ADHD diagnosis, and ADHD type if reported. Results indicate that after controlling for the age of the child and ADHD diagnosis, there were no significant differences between ethnic groups in beliefs of behavioral treatment, $F(1,116)=0.042, p=.837$. However, significant group differences still remain after controlling for ADHD type $(p=.003)$ and child's gender $(p<.001)$.

In regard to parental views of ADHD as a biopsychosocial disorder, only $7.0 \%$ of parents $(n=8)$ scored high on both beliefs of biological/genetic causes and beliefs of behavioral/psychological causes ( $M=$ 3.04), which suggests that they view causes of ADHD from a biopsychosocial perspective. Out of these eight parents, five were of ethnic majority, and three were of ethnic minority. All eight parents reported having a child diagnosed with ADHD. In regard to treatment, $46.2 \%$ of parents $(n=55)$ scored high on both beliefs of medication treatment and beliefs of behavioral treatment, which suggests that these parents might have a strong view on a biopsychosocial treatment approach for ADHD $(M=$ 3.38). Out of the 55 parents, 34 parents were of ethnic majority, and 21 were of ethnic minority. In addition, 24 of out of the 55 parents who believe in the biopsychosocial treatment approach have children without ADHD. All eight parents who scored high on beliefs of biopsychosocial causes of ADHD reported high scores on beliefs of biopsychosocial treatment $(M=3.34)$.

A content analysis was performed on the two openended questions. A majority of parents (40.0\%) reported (in many instances the exact phrase) that there was a "chemical imbalance in the brain." Another prevalent response included ADHD having a genetic component
(22.7\%). Other parents suggested environmental factors or stressors (e.g., family divorce) allowed children to develop ADHD, though the data primarily showed that a majority of the responses regarding causes of ADHD was either biologically or genetically based. Of the responses, $16.0 \%$ suggested a combination of factors that relate to both biological and environmental factors. Last, 23 parents (19.3\%) reported not knowing the causes of ADHD; however, it was reported only by parents of children who did not have ADHD.

\section{Treatment Preferences}

Parents largely reported they would prefer combined treatment (both medication and behavioral treatment or counseling) for their child with ADHD (53.8\%), even those parents who did not have a child with ADHD. This percentage was somewhat comparable to the $46.2 \%$ of parents who scored high on beliefs of biopsychosocial treatment, based on the quantitative data. Reasons for selecting this treatment include its effectiveness and that the treatment targets different areas of concern (e.g., helping the brain to calm down, so the children can learn strategies from a counselor to help them in classroom). There were more parents who preferred only counseling (24.4\%) than only medication (17.6\%). For parents who had a child with ADHD, the most frequently reported reason for why parents preferred only medication was that their child's doctor recommended it. In addition, another reason for choosing medication over counseling was that the parents believed that counseling was ineffective. For example, one parent reported, "Counseling was taking too long and was not working for my child, but the medication was helping him focus in school." Those who preferred only counseling, however, reported negative experiences with medication because of its stigma and side effects. One parent reported, "The medication made my child like a zombie. .. . I took him off it." For parents who have a child without ADHD, slightly more parents preferred only counseling (16.8\%) than only medication (12.7\%) based on common beliefs that medication has "too many side effects." Only $5.0 \%$ reported not using any of the aforementioned treatments. Table 2 provides the number and percentage of parents who reported specific treatment preferences separated by ethnicity and ADHD diagnosis.

A multinomial logistical regression was used to determine whether parental beliefs of ADHD and treatment predicted their choice of treatment. There was a significant relationship between beliefs of biological/genetic causes of ADHD and preference for only medication treatment ( $p=.037)$. However, beliefs of behavioral/psychological causes of ADHD did not significantly predict parents' 
Table 2 Number and Percentage of Parents' Treatment Preferences by Ethnicity and ADHD Diagnosis

\begin{tabular}{|c|c|c|c|c|c|c|c|c|c|c|}
\hline & \multicolumn{2}{|c|}{$\begin{array}{c}\text { Only } \\
\text { Medication }\end{array}$} & \multicolumn{2}{|c|}{$\begin{array}{c}\text { Only } \\
\text { Counseling }\end{array}$} & \multicolumn{2}{|c|}{$\begin{array}{l}\text { Combined } \\
\text { Treatment }\end{array}$} & \multicolumn{2}{|c|}{$\begin{array}{l}\text { None of } \\
\text { the Above }\end{array}$} & \multicolumn{2}{|c|}{ Total } \\
\hline & $n$ & $\%$ & $n$ & $\%$ & $n$ & $\%$ & $n$ & $\%$ & $n$ & $\%$ \\
\hline Ethnic majority (Caucasian) & 8 & 12.5 & 11 & 17.2 & 45 & 70.3 & 0 & 0.0 & 64 & 100.0 \\
\hline Child with ADHD & 4 & 6.2 & 1 & 1.5 & 23 & 35.9 & 0 & 0.0 & 28 & 43.8 \\
\hline Child without ADHD & 4 & 6.2 & 10 & 15.6 & 22 & 34.4 & 0 & 0.0 & 36 & 56.2 \\
\hline Ethnic minority & 12 & 21.8 & 18 & 32.7 & 19 & 34.5 & 6 & 10.9 & 55 & 100.0 \\
\hline Child with ADHD & 9 & 16.3 & 8 & 14.5 & 13 & 23.6 & 0 & 0.0 & 30 & 54.5 \\
\hline Child without ADHD & 3 & 5.5 & 10 & 18.2 & 6 & 10.9 & 6 & 10.9 & 25 & 45.5 \\
\hline African American & 7 & 12.7 & 8 & 14.5 & 14 & 25.4 & 4 & 7.2 & 33 & 60.0 \\
\hline Latino American & 5 & 9.1 & 10 & 18.2 & 5 & 9.1 & 2 & 3.6 & 22 & 40.0 \\
\hline Total & 20 & 16.8 & 29 & 24.4 & 64 & 53.8 & 6 & 5.0 & 119 & 100.0 \\
\hline
\end{tabular}

preference for only counseling $(p=.440)$. Beliefs of biological/genetic causes of ADHD $(p=.084)$ and beliefs of behavioral/psychological causes of $\operatorname{ADHD}(p=.060)$ did not significantly predict combined treatment. However, parents of children with ADHD $(n=8)$ who rated ADHD as having biopsychosocial causes rated biopsychosocial services more positively than parents who did not attribute ADHD as a biopsychosocial disorder. Beliefs of medication treatment significantly predicted preference for only medication $(p=.012)$ and combined treatment methods $(p=.033)$, whereas beliefs of behavioral treatment significantly predicted preference for only counseling $(p=.018)$.

\section{Discussion}

The present study tested four research questions relating to ethnic differences relating to parental beliefs about ADHD, attitudes toward efficacious treatment approaches, and how these beliefs influence treatment choice. Results did not support previous research findings on how ethnic minority parents have different beliefs about causes of ADHD compared with Caucasian parents. Findings suggest that ethnic minority parents may have increased knowledge about the causes ADHD, taking into account the importance of biological factors and thus closing the knowledge gap between ethnic minority parents and Caucasian parents about the disorder. Qualitative data also supported these findings as many parents (40\%) reported that the primary cause of ADHD is because of a "chemical imbalance in the brain," which is known to be one of the characteristics of the disorder. However, parents of children with ADHD rated biological or genetic causes more positively than did parents of children without ADHD. This finding supports studies that have demonstrated those who have not experienced
ADHD are less likely to understand the biological causes of the disorder compared to those who have children with ADHD (Taylor \& Leitman, 2003).

The current literature and research exploring the etiology of ADHD emphasized a biopsychosocial model to understanding the disorder (Rapport et al., 2001); however, only $7 \%$ to $16 \%$ of parents were aware that ADHD is caused by a combination of biological and psychological factors based on the qualitative and quantitative data of the questionnaire. Even though only a small percentage of parents in this study attributed symptoms of ADHD from this biopsychosocial perspective, group differences appeared minimal. Despite the lack of ethnic differences of how parents explain the etiology of ADHD, ethnic majority parents are more likely to pursue both biopsychosocial services (e.g., medication and behavioral treatment) than are ethnic minority parents (Yeh et al., 2004). This finding suggests that additional social, educational, and financial factors may contribute to the understanding of how ethnic minority parents seek treatment for their child with ADHD.

A small percentage of parents who have a child without ADHD reported causes of ADHD that have not been supported by literature. For example, some parents believed excessive television viewing or computer playing can cause ADHD. In addition, close to one fifth of parents reported that they did not know what causes ADHD. However, those who reported this did not have a child with ADHD. Therefore, parents with children who have ADHD may be more knowledgeable about the etiology and treatments of ADHD than parents who do not have children with the disorder (Taylor \& Leitman, 2003).

There were no significant group differences found in regard to how each group views the acceptability of medication treatment. This finding suggests that stigmas 
or negative attitudes about medication treatment have been gradually fading within the past decade. However, African American and Latino parents still rated behavioral interventions significantly higher than did Caucasian parents. Ethnic minority parents tend to favor behavioral interventions more than Caucasian parents as their preferred method of treatment for ADHD (dosReis et al., 2003; Perry et al., 2005). When controlling for age, gender, and ADHD type, the child's age was the only factor that influenced treatment preference. Therefore, parents considered the relevance of their child's age when deciding on specific treatments. Because parents can be reluctant to administer medication to a child at a very young age, some parents would consider pursuing an alternative method of treatment, such as behavioral intervention, that would be perceived as safer yet produce the same positive effects.

Parents who believe that ADHD is a disorder that biologically or physiologically affects the brain may conclude that medication would "calm" the brain to help the child's behaviors. According to the content analyses, some parents realized that the environmental or psychological factors can exacerbate symptoms of ADHD; however, parental beliefs about behavioral or psychological causes of ADHD did not significantly predict parents' preference for behavioral treatment. One reason for this finding could be that even though parents rated behavioral treatment more positively than medication, many parents, especially ethnic minority parents, are becoming more aware and knowledgeable about the important biological components of ADHD. Therefore, medication was reported more often as part of the treatment regimen because of the increased emphasis of the role of biology in children with ADHD. Reasons for choosing solely behavioral interventions as the parents' method of treatment appeared only if an alternative (i.e., medication) was not deemed suitable for their child, including consideration of the child's age, the medication's lack of effectiveness, or its negative side effects (dosReis et al., 2003).

Parents who held favorable beliefs about medication treatment would likely prefer medication as their treatment choice for their child (dosReis et al., 2003). In addition, they would choose combined treatment methods as some parents believe that children need to learn skills to manage their difficulties with attention and/or their hyperactivity. Parents who hold positive beliefs about behavioral treatment would likely choose only behavioral interventions but not combined treatment methods. Because parents who favor solely behavioral treatment tend to have negative attitudes toward medication, they would not likely choose medication as part of the child's treatment regimen.
In conclusion, this research study provides information from a relatively diverse sample of parents exploring their beliefs about the etiology of ADHD and attitudes toward various efficacious treatment approaches. Ethnic minority families appear to be more informed about causes of ADHD now than a decade ago. Because ethnic group differences were apparent in relation to views about various treatments, it is important for researchers and practitioners to recognize that parents have their own personal beliefs about mental health disorders, which can indirectly influence treatment decisions. Both medication and behavioral interventions have been proven to be efficacious; however, parents are likely to choose one over another based on a child's age, prior experiences with a specific treatment, and attitudes toward the treatment. For future research, other factors including economic status (e.g., health insurances, financial resources), community and social networks, and service characteristics (e.g., bilingual clinicians, culturally sensitive services) should be considered as they may serve as potential barriers for many families to receive appropriate services.

\section{Limitations of the Study}

There are a number of limitations that deserve consideration. Recruiting an adequate number of parents of African American and Latino parents who have a child with ADHD proved difficult because there was not a large sample that fit the criteria at the recruitment sites. There were demographic and geographic limitations to the study considering that all participants were recruited from the mid-Michigan area and are not likely representative of a national sample. Because of the small sample size, it would be hard to generalize these findings to the ethnic minority population. Increasing the number of participants would increase the power of this study as well as lower the effect size. There was also little control for confounding variables, particularly the socioeconomic status and educational level of parents. One other reason why ethnic minority families may not prefer certain ADHD services is the cost of services. Some parents may not be able to afford medication or participate in behavioral treatment programs. Further research will hopefully control for these factors.

Another limitation is the recruitment of Latino parents. Because this study and the questionnaire were written entirely in English, Latino parents who were proficient in reading and writing in English were primarily recruited for this study. Thus, the sample of Latino parents who participated in this study might not accurately reflect Latino parents who speak Spanish as their first or only language. Fortunately, no participants withdrew from the study because of language barriers. 
In addition, it is difficult to determine the directionality in the relations between parents' beliefs and attributions and their treatment experiences. Parents' cognitions may drive treatment choices or may reflect the experiences that parents have had with various treatments for their child with ADHD. Despite these limitations, this study provides preliminary both quantitative and qualitative data for researchers and practitioners to understand beliefs and attitudes of ethnic minority parents regarding ADHD and treatment.

\section{Implications for Practitioners}

The significant increase in children diagnosed with ADHD intensifies the need for parents to be informed and aware of the treatment options for ADHD symptoms. Ultimately, these parents make the final decision for the assessment and treatment of ADHD for their children. Considering that parents obtain a lot of their resources and information about ADHD from physicians and school professionals (e.g., school psychologists), practitioners are in a position to provide education to families about symptoms and interventions for children with ADHD. During parent interviews, it is important to listen to how parents view and explain their child's behaviors and also how they attempted to manage them. However, considering that parents may bring different cultural beliefs to the treatment context, discrepancies between the beliefs of the parents and clinician may exist. Therefore, practitioners must respect and build on their own cultural knowledge as well as the families' strengths to determine together what services would be acceptable for all parties involved. In addition, future research should continue to examine the specific role of explanatory etiologies in service utilization patterns and treatment effectiveness for families with ADHD.

\section{References}

Bailey, R. K. (2005). Diagnosis and treatment of attention-deficit/ hyperactivity disorder (ADHD) in African-American and Hispanic Patients. Journal of the National Medical Association, 97, 3s-4s.

Bailey, R. K., \& Owens, D. L. (2005). Overcoming challenges in the diagnosis and treatment of attention-deficit/hyperactivity disorder in African Americans. Journal of the National Medical Association, 97, 5s-10s.

Brown, R. T. (2005). Recent advances in pharmacotherapies for the externalizing disorders. School Psychology Quarterly, 20, 118-134.

Bussing, R., Gary, F. A., Mills, T. L., \& Garvan, C. W. (2003). Parental explanatory models of ADHD: Gender and cultural variations. Social Psychiatry \& Psychiatric Epidemiology, 38, 563-575.

Bussing, R., Zima, B., \& Belin, T. (1998). Differential access to care for children with ADHD in special education programs. Psychiatric Services, 49, 1226-1229.

dosReis, S., Zito, J. M., Safer, D. J., Soeken, K. L., Mitchell, J. W., \& Ellwood, L. C. (2003). Parental perceptions and satisfaction with stimulant medication for attention-deficit hyperactivity disorder. Developmental and Behavioral Pediatrics, 24, 155-162.

Eiraldi, R. B., Mazzuca, L. B., Clarke, A. T., \& Power, T. (2006). Service utilization among ethnic minority children with ADHD: A model of help-seeking behavior. Administration and Policy in Mental Health, 33, 607-622.

Johnston, C. (2001). ADHD Beliefs and Attitudes Scale. Unpublished scale. [Available from Dr. Johnston at cjohnston@psych .ubc.ca]

Johnston, C., Seipp, C., Hommersen, P., Hoza, B., \& Fine, S. (2005). Treatment choices and experiences in attention deficit and hyperactivity disorder: Relation to parents' beliefs and attributions. Child: Care, Health and Development, 31, 669-677.

Majewicz-Hefley, A., \& Carlson, J. (2007). A meta-analysis of combined treatments for children diagnosed with ADHD. Journal of Attention Disorders, 10, 239-250.

Perry, C. E., Hatton, D., \& Kendall, J. (2005). Latino parents' accounts of attention deficit hyperactivity disorder. Journal of Transcultural Nursing, 16, 312-321.

Rapport, M., Chung, K., Shore, G., \& Isaacs, P. (2001). A conceptual model of child psychopathology: Implications for understanding attention deficit hyperactivity disorder and treatment efficacy. Journal of Clinical Child Psychology, 30, 48-58.

Rostain, A., Power, T., \& Atkins, M. (1993). Assessing parents' willingness to pursue treatment for children with attention-deficit hyperactivity disorder. Journal of the American Academy of Child and Adolescent Psychiatry, 32, 165-181.

Rothe, E. M. (2005). Considering cultural diversity in the management of ADHD in Hispanic Patients. Journal of the National Medical Association, 97, 17s-25s.

Stroh, J., Frankenberger, W., \& Cornell-Swanson, L. (2005). Attention Deficit Hyperactivity Disorder and Treatment Survey. Unpublished scale. [Available from Dr. Frankenberger at frankewr@uwec. edu]

Taylor, H., \& Leitman, R. (2003). Barriers to the diagnosis and treatment of ADHD among African-American and Hispanic children. Health Care News, 3, 111-114.

Yeh, M., Hough, R. L., McCabe, K., Lau, A., \& Garland, A. (2004). Parental beliefs about the causes of child problems: Exploring racial/ethnic patterns. Journal of the American Academy of Child and Adolescent Psychiatry, 43, 605-612.

Andy V. Pham, M.A. is a doctoral candidate in the School Psychology program at Michigan State University. His research interests include assessment and intervention of neurodevelopmental disorders such as learning disabilities, ADHD, and autism spectrum disorders.

John S. Carlson, PhD is an Associate Professor of School Psychology at Michigan State University. His research interests include evidenced-based interventions with specific attention to issues of effectiveness and cost-benefit analyses, school psychopharmacology, social anxiety/selective mutism, and externalizing behavior disorders.

John F. Kosciulek, PhD is a Professor of Rehabilitation Counseling at Michigan State University. His research interests include research ethics and methodology, vocational rehabilitation, and school-to-career transition of students with and without disabilities. 